\title{
DIT DE L'HERBERIE
}

\author{
Miroslava Novotná
}

\begin{abstract}
The goal of this paper is to present the role of the quack doctor by Rutebeuf in the history of the French comedy and his possible influence on the Czech literature. We try to unveil a heart of laughter and relationships in the medieval society on the basis of the analyzed work. Considering the fact that the Rutebeuf's work is mainly connected to events from his period of time and as these events have a lot in common with the current society, we focus principally on the elements that make the Rutebeuf's work still present. "Rutebeuf writes to earn his living, mostly by command. Nevertheless his poesy creates an illusion of constant flow of words and even the most vigilant critics believe that his lines reflect his life and his moral standards. (Rutebeuf, CEuvre complète, Préface de Michel Zink, Paris, Lettres gothiques 2005, p. 23).
\end{abstract}

Keywords: Rutebeuf; literature; theatre; Middle Age.

Résumé : Notre intervention se concentrera sur le rôle du Dit de l'Herberie de Rutebeuf dans l'histoire de la comédie française et sur son influence dans la littérature tchèque. Nous essaierons de dévoiler des facteurs du rire et des relations dans la société médiévale des ouvrages analysés. Comme l'œuvre de Rutebeuf est, pour une large part, liée à certains événements de son temps et comme ces événements ont quelque chose de commun avec notre société contemporaine, nous soulignerons également cet effet qui rend l'œuvre de Rutebeuf toujours actuelle : «Rutebeufécrit pour vivre, et le plus souvent sur commande. Mais ses poèmes créent l'illusion de l'épanchement au point que les critiques les plus avertis y cherchent un reflet de sa vie et de son itinéraire moral. » (Rutebeuf, CEuvre complète, Préface de Michel Zink, Paris, Lettres gothiques 2005, p. 23).

Mots clés : Rutebeuf ; littérature ; théâtre ; Moyen Âge.

\section{Introduction}

Le but du présent article est de se concentrer sur l'évolution du personnage de l'herboriste (ou de l'herbier dans certains textes du Moyen Âge des pièces liturgiques aux pièces profanes telles que Dit de l'Herberie de Rutebeuf. Ce personnage possède sa propre évolution, il a même ses doubles dans les autres " performances » du théâtre européen.

Nos réflexions s'appuient sur l'étude de treize textes qui remontent à l'époque située entre les $\mathrm{XI}^{\mathrm{e}}$ et $\mathrm{XIV}^{\mathrm{e}}$ siècles. 


\section{Un personnage spécifique}

Le personnage du marchand ou de l'herbier ${ }^{1}$ et par la suite du faux médecin ne quitte pas la scène du théâtre européen depuis ses origines jusqu'à nos jours. L'herbier ou le marchand qui vend des simples et des aromates, dont les trois Marie ont besoin pour embaumer le corps de Jésus-Christ, n'est pas mentionné dans les Evangiles explicitement, mais son existence est déductible à partir du verset suivant : "Quand le sabbat fut passé, Marie de Magdalena, Marie, mère de Jacques, et Salomé achetèrent des aromates pour aller l'embaumer. $»^{2}$

Dans les pièces liturgiques, le marchand était presque le seul personnage profane qui jouait le rôle d'élément indispensable à la suite des scènes bibliques. Dans l'Époux (Sponsus), ou Drame de l'Époux, ou Mystère des vierges sages et des vierges folles, ${ }^{3}$ une des premières pièces écrites en latin et complétées par la didascalie en dialecte occitan, nous rencontrons des marchands qui auraient pu sauver les vierges folles de la damnation éternelle s'ils avaient eu de l'huile à leur disposition. À côté des cinq vierges sages, des cinq vierges folles et de personnages uniques tels que Jésus ou l'Époux on peut signaler la présence d'une quantité indéterminée de marchands. De cette façon, ils créent un espace de transition entre deux univers : celui des gens qui ont un comportement différent dans la vie courante et dans le cadre de leur foi et celui de ceux qui vivent de la même façon dans leurs sphères quotidiennes et religieuses. Le rôle transitif est tenu par le personnage du marchand ou de l'herbier dans les jeux pascaux.

Jarmila Felzmann Veltrusky ${ }^{4}$ cherche dans ses études une réponse à la question relative à l'importance du rôle de l'herbier dans les drames liturgiques liés aux Pâques : ces pièces étaient jouées à la fin des Matines, chantées par toute la communauté. Les moines ou les religieuses, qui jouaient les trois Marie, se levaient et quittaient leurs bancs et se dirigeaient vers le Saint-Sépulcre. Ils étaient obligés d'interrompre leur chemin pour se procurer des accessoires nécessaires pour embaumer le corps de Jésus-Christ.

Gustave Cohen ${ }^{5}$ présente la description de la mise en scène des pièces liturgiques pascales organisées et jouées à l'intérieur de la cathédrale de Tours :

\footnotetext{
1 Le mot herbier est employé par Rutebeuf et par Michel Zink. Le dernier emploie également le mot marchand de simples, les autres auteurs marchand. En français moderne il s'agit de l' " herboriste ». Dans le présent texte, nous utilisons le herbier pour conserver le caractère « médiéval ».

$2 C f$. Veltrusky, J. F., « Postava Mastičkáře ve středověkém náboženském divadle » in. Divadelní revue, année 13, numéro 4, Prague 2002, p. 4, et $c f$. la traduction œcuménique tchèque de la Bible, 1995. Toutes les références renverront à cette édition, abrégée comme suit : Postava Mastičkáře, suivi du numéro de la page.

3 Drame liturgique anonyme bilingue (40 vers en occitan, 47 vers en latin) du XIe siècle qui provient de l'abbaye Saint-Martial de Limoges. Première manifestation de la dramaturgie, ce texte, qui s'inspire de la parabole des vierges sages et des vierges folles (Évangile de Matthieu, XXV,1-13), est composé de strophes de types variés, avec ou sans refrain, accompagnées d'un jeu de mélodies.

4 Jarmila Felzmannová Veltruský (22/6/1937 à Prague). Son père était le premier secrétaire de l'Ambassade de Tchécoslovaquie à Londres qui, depuis 1948, séjourna en Angleterre. Jarmila F. Veltruský a vécu la majorité de sa vie en Angleterre et en France. Elle a traduit l'Herboriste tchèque en anglais et elle est l'auteure du livre Mastičkár̆, A Sacred Farce from Medieval Bohemia (1985). Cf. Stehlíková, E. „Dáma se zvláštním jménem a divadlo českého středověku“ in Divadelní revue, année 13, numéro 4, Prague 2002, p. 3.

5 Cf. Hue, D. ; Cycle de Pâques, Version informatisée [en ligne], http://www.sites.univ-rennes2.fr/celam/ cetm/liturg/paques.htm [consulté le 1er mai 2010]. Toutes les références renverront à cette édition, abrégée comme suit: Cycle de Pâques, suivi du numéro de la page.
} 


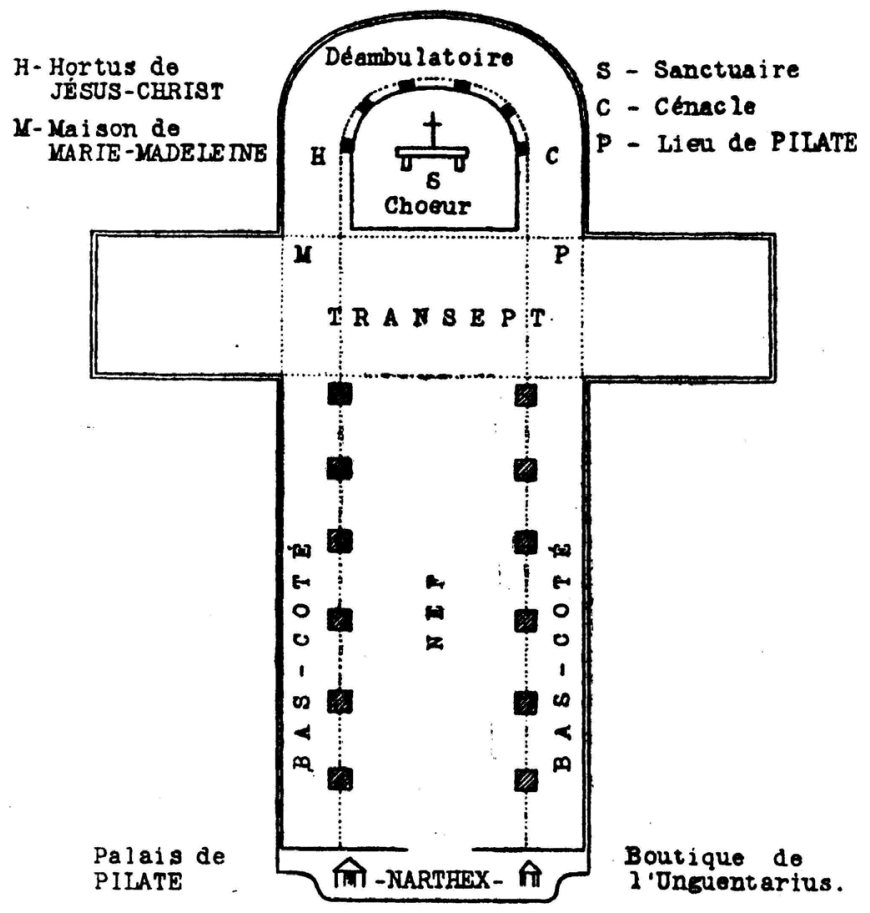

L'organisation de l'espace est inclue dans la didascalie de la pièce et même dans les vers latins du Drame pascal de la Résurrection de Marmoutier (Tours) du XII siècle. $^{6}$

Le fait que le marchand a son statut spécifiquement marqué qui se trouve à la frontière entre le monde fictif et celui où la représentation se déroule, à la frontière entre l'univers liturgique et l'univers profane, est le point de départ primordial pour l'évolution autonome du personnage de l'herbier.

Nous avons eu à notre disposition treize textes où le(s) marchand(s) ou l'herbier jouent les rôles principaux. J. F. Veltrusky parle dans une de ses études de quatorze textes médiévaux, si l'on accepte les pièces françaises, des textes allemands, espagnols et tchèques. Elle mentionne l'existence de textes du théâtre médiéval russe et balkanique et montre l'effet appelé par Roman Jakobson effet « des identités mystérieuses » en se référant aux scènes de l'herbier bien qu'elles apparaissent dans des époques et des lieux différents. ${ }^{7}$ Parmi nos treize textes étudiés et les quatorze textes présentés par J. F. Veltrusky il n'y en a que 6 identiques étudiés par Veltruský et par nous-même : Ludus paschalis de Tours, l'Herbier de Rutebeuf, l'Herberie en prose, De la goute en l'aine et deux versions de L'Herbier tchèque.

\footnotetext{
Notons encore que l'herboriste était appelé en latin unguentarius, specionarius, mercator, institor, medicus ou tout simplement barbatus; en français marchans ou marcader ou espicier, en allemand kramer, koufmann ou artz, en tchèque mastičkář ou lékař.

7 Cf. Veltruský, J. F., «Staročeský Mastičkář jako klaun i symbol » in Divadelní revue, année 13, numéro 4, Prague 2002, p. 16.
} 


\section{Le marchand « liturgique»}

La scène-clé qui donne naissance au personnage de l'herbier est présentée, comme nous l'avons déjà indiqué, par la nécessité de se procurer des aromates pour embaumer le corps de Jésus-Christ. Dans la Résurrection du Seigneur, texte du $X^{\mathrm{e}}$ siècle, écrit en latin, on ne mentionne qu'une seule fois les trois femmes qui apportent des parfums :

«Aguntur enim haec ad imitationem angeli Sedentis in Monumento atque mulierum cum aromatibus venientium ut ungerent corpus Jhesu. Tout ceci se pratique à l'imitation de l'Ange assis au sépulcre et des femmes venant avec leurs parfums oindre le corps de Jésus. » [Cycle de Pâques, 4]

Le texte latin de l'Office du Saint-Sépulcre à l'usage de Rouen ${ }^{8}$, lequel date du XI ${ }^{\mathrm{e}}$ siècle, présente également un très court dialogue entre l'ange qui garde le Saint-Sépulcre et les femmes qui chantent et s'approchent du tombeau.

Le Drame pascal de la résurrection de Marmoutier (Tours) du XII siècle fait monter sur la scène même deux marchands : cette pièce se compose de seize tableaux ou scènes établies par Gustave Cohen; dans la scène III arrivent trois Marie qui rencontrent un marchand de parfums. Le texte est écrit encore en latin et contient une didascalie assez détaillée. La scène dans laquelle se rencontrent l'ange, les gardes et les Marie est presque complètement consacrée à la description des actions [Cycle de Pâques, 10].

Ensuite, Marie Madeleine apostrophe Dieu en utilisant des métaphores suggestives qui soulignent la grande douleur des trois Marie. Marie Jacobi évoque Jésus-Christ et achève son propos aussi par l'expression de leur grande douleur. Enfin, Marie Salomé met fin à leur scène par le même vers douloureux que ses deux compagnes après avoir mentionné la nécessité d'aller acheter des aromates. A ce moment-là, le marchand commence à parler, il offre directement sans détour un onguent "pour le corps sacré du Seigneur ». Les trois Marie sont sur le point de le payer à un prix exorbitant, mais à ce moment, le deuxième marchand intervient et demande de quel onguent elles ont besoin et en quelle quantité :

$R$ [espondeat] MERCATOR :

Dicite quid vultis.

MARIAE simul respon[deant ] : Balsamum, thus et mirram, Silaloe et aloes.

$R$ [espondeat $]$ MERCATOR : Ecce jam ante vobis sunt omnia ; Dicite quantum vultis emere?

MARIAE simul respondent Quasi centum libras; satis Habemus. Dic nobis Quantum denarios, Domine?

$R$ [espondeat $]$ MERCATOR : Mille solidos potestis Habere. »
Que le MARCHAND réponde :

Dites ce que vous voulez.

Que les MARIES répondent ensemble:

Du baume, de l'encens, de la myrrhe,

Du silaloë et de l'aloès.

Que le MARCHAND réponde :

Voilà tout cela que je vous présente, Dites combien en voulez-vous acheter?

Que les MARIES répondent ensemble:

Environ cent livres ; assez

Nous en aurons. Dis-nous

Combien de deniers, Seigneur.

Que le MARCHAND réponde :

Vous les aurez pour mille sous [d'or]. [Drames liturgiques de la cathédrale de Rouen, 250]

\footnotetext{
8 Cf. Gaste, Armand ; Drames liturgiques de la cathédrale de Rouen, Evreux, 1893, p. 250. Toutes les références renverront à cette édition, abrégée comme suit : Drames liturgiques de la cathédrale de Rouen, suivi du numéro de la page.
} 
Cette scène évoque une autre scène de la Bible : trois rois offrirent à l'enfant Jésus en don : « de l'or, de l'encens et de la myrrhe » $(\mathrm{Mt} 2,11)$ et c'est Nicodème qui apporte « un mélange de myrrhe et d'aloès d'environ cent livres » $(\mathrm{J} 19,39)$ pour l'enterrement de Jésus-Christ. ${ }^{9}$

Le quatrième texte en ancien français que nous avons pu consulter remonte au XII siècle et il a été édité pour la première fois par Achille Jubinal en $1834 .{ }^{10}$ La Résurrection $d u$ Sauveur commence par un prologue qui présente la disposition des lieux évoqués dans la pièce.

Le dernier texte liturgique étudié remonte au XII ${ }^{\mathrm{e}}$ siècle et il est écrit en provençal : La Passion catalane occitane. Ce texte conserve le cliché sacré habituel : la douleur des trois Marie qui viennent à Jérusalem pour trouver le corps du Christ. Le dialogue repose sur l'attente des événements connus et tient le public en haleine par les formules de politesse échangées, par les questions développées, par les réflexions prononcées à haute voix par les personnes sur la scène : Marie Madeleine commence à parler d'un onguent qui ne devrait pas être trop cher ; Marie Jacobi aperçoit un homme qui semble être un marchand. Ce dernier prononce quelques formules de politesse et assure les Marie qu'elles ne partiront pas les mains vides. Il leur montre un seul onguent et fait une des premières allusions au sens de l'odorat dans le théâtre français médiéval : «Vous voyez comme ça sent. » D'abord, il demande « mille marcs d'argent » pour son onguent, mais à la fin il accepte « cent marcs d'argent fin ». Les Marie le remercient et partent pour Jérusalem. ${ }^{11}$

Maria Magdalena

1836 Donas, doncas anem lo comprar

Prec vos per Dieu que non sia re car,

Maria Jacobi

Que nos em tres que be o podem paguar.

Donas, aujat! que so que avem serca,

A mi semla sia tot acabat.

Demandem le aquel senher honrat.

Totas III diseron al marcader

Ai marcader, bel senher, Dieus vos sal

Respon lo marcader

Nos em femnas del rey esperital.

Dousas donas, be poscatz vos venir!

Guaut vos do Dieu, car tant vos vey marir.

Digat mi ades que volet far ni dir.

E guaut torno vostras dolors!

Maria Salome

Senher, aujat : nos em dolentas fort

Per lo senhor que an los Juzieus mort;

E volem ne ab vos aver acort.

Respon lo mercader

Donas queret ades tot quant vulhat,

Car de tot em nos beaparelhat.

Ja no.n iret fadias, si Dieu plat. ${ }^{12}$

Cycle de Pâques ; pp. 10-11.

10 Monmerque, Louis-Jean-Nicolas, Michel, Francisque ; Théâtre français au Moyen Age, Paris, Firmin-Didot, 1839.

${ }_{11}$ Macdonald, Aileen Ann; Passion catalane occitane, Textes littéraires français, Droz 1999, p. 200.

12 Macdonald, Aileen Ann; Passion catalane occitane, Textes littéraires français, Droz 1999, p. 200. 
L'évolution indiquée du personnage de l'herbier montre les différents stades de l'ébauche et du traitement d'un seul protagoniste, mais aussi l'évolution logique et conçue selon les talents différents des auteurs et des conditions dans lesquelles ils pouvaient créer. J. F. Veltrusky présente encore d'autres types de ce personnage intéressant :

- Celui de Ludus paschalis de Vich (XIe ou XII siècle) a des soucis en ce qui concerne les prix de sa marchandise, mais il parle aussi de sa lutte contre la mort et la décomposition; Ludus paschalis du Codex Buranus (XIII ${ }^{\mathrm{e}}$ siècle) met en scène non seulement $l^{\prime}$ herbier, mais aussi sa femme. Dans la Passion provençale (XIV siècle) l'herbier apparaît avec son fils.

- La Marie Jacobi d'Arnould Gréban dans son Mystère de la passion (vers 1450) affirme connaître très bien l'herbier qui vend des onguents. Le marchand offre aux trois Marie du «balsame fin » et de la «myrrhe et de l'aloès », c'est-à-dire des plantes bibliques à propos desquelles il dit :

$$
\begin{aligned}
& \text { "que s'ung roy, prince ou altre gens } \\
& \text { en sont bien enoingts une foiz, } \\
& \text { on les gardera plus d'un mois } \\
& \text { sans quelque putrefaccion. » }
\end{aligned}
$$

- Dans la Palatinus (début du XIV e siècle) écrite en français, apparaît l'herbier-charlatan pareil à celui de Rutebeuf. [Postava Mastičkáře, 5-7]

Il est alors évident que le personnage du marchand ou de l'herbier ou d'un faux médecin est devenu un passage obligé pour les auteurs, pour les jongleurs qui continuaient à créer leurs propres textes.

\section{L'herbier profane}

Le Dit de l'herberie de Rutebeuf n'est pas un texte unique en son genre.

«On connaît une autre Herberie en prose, plus longue que celle de Rutebeuf, dont elle s'inspire, et une pièce en vers à peu près de la même époque intitulée la Goute en l'aine, qui reproduit le boniment d'un médecin. »[Rutebeuf, cuvres complètes, 765 $]^{13}$

Michel Zink souligne, dans une remarque intéressante, l'éloquence proverbiale des marchands de simples au Moyen Age. Selon un traitement édité vers 1271 par la Faculté de Médecine de Paris «la prescription des remèdes fut réservée aux apothicaires tenant boutique (les épiciers) comme aux marchands ambulants (les herbiers) ». [Rutebeuf, œuvres complètes, 765]

L'histoire de l'herbier, soit sous la forme d'une farce comme dans le cas de ses versions tchèques, soit sous la forme d'un monologue comme celui de Rutebeuf, est une pièce autonome avec son propre univers fictif développé selon des intentions spécifiques et selon le talent de chaque auteur, d'après le goût du public et les conditions socioculturelles.

Rutebeuf, qui était obligé d'écrire pour vivre, a fait revivre le personnage du marchand de simples de manière qui fascine encore le public contemporain par sa vivacité et spontanéité, par le courant continu de mots comprenant tous les sentiments possibles. L'auteur emploie de nombreux procédés dramatiques pour tenir éveillé son public : les apostrophes aux spectateurs, les promesses des remèdes miraculeux, les récits persuasifs des cures les plus bizarres, l'énumération de choses rares, extraordinaires, fascinantes, les

\footnotetext{
${ }^{13}$ Zink, Michel, Rutebeuf, œuvres complètes, Classique Garnier, Paris 2005, p. 765. Toutes les références renverront à cette édition, abrégée comme suit : Rutebeuf, œuvres complètes, suivi du numéro de la page.
} 
éléments burlesques incorporés dans des recettes folles, les conseils garantis, les énumérations des lieux et des pays visités, etc. Tout ceci s'entrelace avec de vraies métaphores poétiques, des calembours, des gradations et des anti-gradations, des paronomases ou des effets d'homophonie. Rutebeuf devient auteur-acteur, jongleur, le metteur en scène de son propre " moi », il attire le public par son jeu, il compte sur son attention, il l'introduit dans ses histoires et s'adresse à tout moment à ses spectateurs, il utilise chaque fois un nouveau stratagème pour emporter la conviction. L'herbier présente par exemple par gradations calculées son habileté et ses expériences réussies en citant Madame Trotula, une femme médecin de l'école de Salerne, qui vivait au XI ${ }^{\mathrm{e}}$ siècle, mais la description de la dame convient également pour la mule de notre herbier.

Un peu plus loin, l'acteur invite le public à le suivre en utilisant les mêmes procédés pour convaincre : "Otez vos capuchons, tendez l'oreille, regardez mes herbes que ma maîtresse envoie dans ce pays. " [Rutebeuf, cuvres complètes, 766]

Rutebeuf sait persuader aussi grâce à des énumérations amusantes. Pour donner plus de force à ses expériences, il cite des pays dont les noms créent une chaîne euphonique.

Michel Zink écrit au sujet des connaissances géographiques :

"La géographie du charlatan mêle les nom de lieux réels et imaginaires. Le nom de Salerne [...] était le siège de la plus célèbre école de médecine du monde médiéval. [...] Burienne et Biterne appartiennent à la toponymie des chansons de geste. »[Rutebeuf, ceuvres complètes, 766-767]

Les éléments burlesques sont à l'œuvre dans les passages où l'herbier prétend, en restant très persuasif, être capable de guérir les maladies les plus extraordinaires :

$\begin{array}{ll}\text { «Je vous dis par sainte Marie, } & \text { De toute fièvre, sauf la fièvre quarte, } \\ \text { ce n'est pas le marché aux puces, } & \text { je guéris en moins d'une semaine } \\ \text { mais des produits de qualité. } & \text { à coup sûr ; } \\ \text { J'ai l'herbe qui redresse les bittes } & \text { je guéris aussi de la fistule ; } \\ \text { et celle qui rétrécit les cons } & \text { si haute ou si basse qu'elle soit ; } \\ \text { sans peine. } & \text { je la réduit complètement. » } \\ & \text { [Rutebeuf, œuvres complètes, 771] }\end{array}$

ce qui ne constitue pas, assurément, la liste exhaustive de toute les maladies citées dans le texte. Un passage très suggestif est dédié aux vers dans le corps humain. Dans son récit, Rutebeuf fait s' entrelacer les effets terrifiants et la bizarrerie, il joue avec la curiosité des spectateurs, au moyen d'effets de surprise et de la gradation de ses arguments. Le récit culmine avec une recette qui paraît vraiment peu fiable.

«Il y a des gens qui me demandent d'où viennent les vers. Je vous informe qu'ils viennent de divers aliments réchauffés et des vins qui ont pris le goût de tonneau et qui ont la pousse, et ils se rassemblent dans le corps à cause de la chaleur er des humeurs ; [...] Et c'est pourquoi les vers viennent dans le corps, montent jusqu'au cœur er font mourir d'une maladie qu'on appelle mort subite. Signez-vous : que Dieu vous en protège tous et toute !

Pour guérir de la maladie des vers, la meilleure herbe qui soit dans les quatre parties du monde - vous la voyez de vos yeux, vous la foulez de vos pieds - c'est l'armoise ! » [Rutebeuf, auvres complètes, 775-777]

Il est vrai que le Dit de l'herbier est un texte suggestif, amusant, fou et persuasif en même temps. Nous sommes tout à fait d'accord avec Michel Zink qui constate :

« La poésie de Rutebeuf porte. Elle porte par sa vigueur persuasive et polémique. Mais elle porte aussi le lecteur par une sorte de recherche de la fascination par la facilité qui en fait une 
poésie du flot et du flux : le rythme à la fois satisfaisant et dégingandé. » [Rutebeuf, cuvres complètes, 33]

Il est alors compréhensible que le texte de Rutebeuf ait eu une forte influence sur les jongleurs de son époque et les époques suivantes. L'Herberie en prose et De la goute en l'aine en sont témoins. Le charlatan du premier texte n'est pas aussi « savant » que celui de Rutebeuf, mais il est également éloquent et roublard. Il commence son récit par cinq conseils sur ce qu'il faut faire pour mettre une femme enceinte. Lui aussi, il invite le public à regarder et à admirer les herbes, les racines, les bestes fauvaiges dont il fait les onguents pour les malades, les bleciez et les navrez. Il a passé par maints pays, Sologne inclus, il sait guérir le mal de dents, mais aussi rajeunir les gens.

Le motif du renouvellement de la force et de la jeunesse avec le motif de la mort et de la résurrection apparaissent dans d'autres scènes de plusieurs textes en Europe, ceux de Bohême inclus.

Même l'herbier du texte en prose possède son herbe maîtresse, mais il s'agit cette fois de l'endive. L'herbier a dû même lutter contre un monstre pour la gagner. Ce monstre Herbelin de Saint-Pol « fu moitié home et moitié feme, E la tierce part chevax. » ${ }^{14}$ Le dialogue entre Herbelin et l'herbier évoque, comme Achile Jubinal le note, l'un des dialogues de la pièce Le Pédant joué de Cyrano de Bergerac.

Pour finir cette petite approche sur les herbiers dans les textes dramatiques médiévaux français, il faut encore attirer l'attention sur un court monologue " $d^{\prime}$ un bon mire $e^{15}$ de Salerne ». Après avoir apostrophé le public et présenté ses bonnes qualités, le «médecin » récite sa recette burlesque à propos de la préparation d'un remède efficace : il faut mêler la corde d'un pendu, la queue d'un lièvre, la laine d'une chèvre, le miel amer, le foie doux, l'aveinière d'une truie, du blanc du cul d'un noir chaudron, le cinquième pied d'un mouton. Ce remède, il faut le prendre à jeun pour être guéri immédiatement.Malgré son caractère convaincant, cette recette voulait faire rire et le rire est l'une des conditions primordiales humaines pour vivre et survivre. Les deux versions tchèques ont le même but. De plus, le personnage de la farce médiévale trouvera une autre vie mouvementée et pleine d'aventures et de mystères dans un roman de Jan z Hvězdy, ${ }^{15}$ écrivain de l'époque de la renaissance nationale. Sous le nom de l'herbier Severin se cache un brave chevalier appelé le Roland de Bohême. Il lutte contre les Allemands carinthiens à l'époque de Jean de Luxembourg et Elisabeth des Přemyslides. Mais ceci est une autre histoire.

\section{Bibliographie}

Gaste, Armand (1893), Drames liturgiques de la cathédrale de Rouen, Evreux.

HeERs, Jacques (1983), Fêtes des fous et carnavals, Paris : Fayard.

Jubinal, Achille (1870), CEuvres complètes de Rutebeuf.

Macdonald, Aileen Ann (1999), Passion catalane occitane, Textes littéraires français, Genève : Droz.

Monmerque, Louis-Jean-Nicolas, Michel, Francisque (1839), Théâtre français au Moyen Age, Paris : Firmin-Didot.

StehLíKovÁ, Eva (2002), « Dáma se zvláštnímjménem a divadločeského středověku », in Divadelní revue, année 13, Praha : numéro 4, p. 3.

\footnotetext{
${ }^{14}$ Mire signifie médecin.

15 Jan z Hvězdy, pseudonyme de l'écrivain tchèque Jan Jindřich Marek (1803-1853) qui a écrit le roman Mastičkár (L'Herbier), Prague, B. Kočí, 1926.
} 
Veltrusky, Jarmila F. (2002), « Postava Mastičkáře ve středověkém náboženském divadle », in. Divadelní revue, année 13, Praha : numéro 4, pp. 4-12.

Z HvĚZdy, Jan (1926), Mastičkář, Praha : B. Kočí.

ZINK, Michel (2005), Rutebeuf, œuvres complètes, Paris : Classique Garnier.

BRAHMA, Kumaris, La condition humaine. Version informatisée [en ligne], http:// fr.answers.yahoo.com/question/index?qid=20080730080708AA1gLMl, [consulté le 9 mai 2010].

Hue, D. ; Cycle de Pâques, Version informatisée [en ligne], http://www.sites.univrennes2.fr/celam/cetm/liturg/paques.htm, [consulté le 1er mai 2010].

Miroslava Novotná

Katedra francouzského jazyka a literatury

Pedagogická fakulta

Masarykova univerzita

Poř́ććí 9

60300 Brno

République tchèque

mir.novotna@gmail.com 